\title{
Pensamiento algebraico temprano de estudiantes de educación primaria (6-12 años) en problemas de generalización de patrones lineales
}

\author{
Ma Luz Callejo, Universidad de Alicante (España) \\ Álvaro García-Reche, Universidad de Alicante (España) \\ Ceneida Fernández, Universidad de Alicante (España)
}

Recibido el 11 de febrero de 2015; aceptado el 8 de Enero de 2016

Pensamiento algebraico temprano de estudiantes de educación primaria (6-12 años) en problemas de generalización de patrones lineales.

\section{Resumen}

Este trabajo identifica características del pensamiento algebraico en alumnos de educación primaria (6 a 12 años) cuando resuelven un problema de generalización de patrones lineales con una sucesión de figuras. Los resultados indican que el pensamiento algebraico se puede iniciar con la coordinación de la estructura espacial y numérica puesta de manifiesto por el uso de representaciones gráficas para hacer un recuento. El cambio de una representación gráfica a una estrategia funcional supone un salto cualitativo ya que implica apoyarse en una figura particular para explicar los cálculos usando deícticos espaciales, o dar explicaciones sobre las figuras en general usando la indeterminación.

Palabras clave. Pensamiento algebraico; generalización; patrones lineales; estrategias; resolución de problemas

Pensamento algébrico precoce de estudantes do ensino básico (6-12 anos) em problemas de generalização de padrões lineares

\section{Resumo}

Este trabalho identifica características do pensamento algébrico em alunos de ensino básico (6 a 12 anos) quando resolvem um problema de generalização de padrões lineares com uma sucessão de figuras. Os resultados indicam que o pensamento algébrico se pode iniciar com a coordenação da estrutura espacial e numérica evidenciada pelo uso de representações gráficas para fazer uma contagem. A passagem de uma representação gráfica a uma estratégia funcional exige um salto qualitativo, uma vez que implica apoiar-se numa figura particular para explicar os cálculos usando deiticos espaciais, ou dar explicações sobre as figuras em geral usando a indeterminação.

Palavras chave: Pensamento algébrico; generalização; padrões lineares; estratégias; resolução de problemas

Para citar: Callejo, M.J., García-Reche, A., \& Fernández, C. (2016). Pensamiento algebraico de estudiantes de educación primaria (6-12 años) en problemas de generalización de patrones lineales. Avances de Investigación en Educación Matemática, 10, 5 - 25.

(C) Sociedad Española de Investigación en Educación Matemática (SEIEM). www.seiem.es 
Early algebraic thinking of primary school pupils (6-12 years) related to linear patterns generalizing problems.

\section{Abstract}

This paper identifies characteristics of primary school pupils (6-12 years) algebraic thinking when solving a linear patterns generalizing problem with a succession of figures. Results show that algebraic thinking starts with the coordination of spatial and numerical structures. It is evidenced in students' use of graphical representation to do a recount. The change from a graphical representation to a functional strategy implies a qualitative step since these students relied on a particular figure to explain their calculations using spatial deictic or they give general explanations of figures using the indeterminacy.

Key words. Algebraic thinking; generalization; linear patterns; strategies; problem solving.

Pensée algébrique précoce d'étudiants d'éducation primaire (6-12 ans) dans des problèmes de généralisation de patrons linéaires.

\section{Résumé}

Ce travail identifie des caractéristiques de la pensée algébrique chez des élèves d'éducation primaire (6-12 ans) quand ils résolvent un problème de généralisation de patrons linéaires avec une succession de figures. Les résultats indiquent que la pensée algébrique peut s'initier avec la coordination de la structure spatiale et numérique mise en évidence par l'usage de représentations graphiques pour faire un comptage. Le changement d'une représentation graphique à une stratégie fonctionnelle suppose un saut qualitatif puisque cela implique s'appuyer sur une figure en particulier pour expliquer les calculs en utilisant des déictiques spatiaux, ou donner des explications sur les figures en général en utilisant l'indétermination.

Mots clés : Pensée algébrique; généralisation; patrons linéaires; stratégies ; résolution de problèmes.

\section{Introducción}

Un foco importante de la investigación en educación matemática en los últimos años ha sido el desarrollo del pensamiento algebraico en los primeros niveles de enseñanza (Radford, 2014), que apoya la introducción del Algebra temprana en el currículum de educación primaria (Cai \& Knuth, 2011; Carraher \& Schliemann, 2007; Godino, Aké, Gonzato \& Wilhelmi, 2014; Socas, 2011). Con esta perspectiva se propone organizar la enseñanza de la aritmética y del álgebra sin saltos ni rupturas, tratando de que haya una continuidad sin necesidad de introducir nuevos tópicos.

Kieran (2004, p. 49) indica que el pensamiento algebraico en las primeras etapas escolares "debería incluir el desarrollo de formas de pensar sobre la relación entre cantidades, la identificación de estructuras, el estudio del cambio, la generalización, la resolución de problemas, la modelación, la justificación, la prueba y la predicción".

Centrándonos en la generalización, ésta ha sido conceptualizada desde diferentes puntos de vista. Según Polya (1965) "la generalización consiste en pasar del examen de un conjunto limitado de objetos al de un conjunto más extenso que incluya al conjunto limitado" (p. 97). Para Dreyfus (1991) "la generalización consiste en derivar o inducir desde lo particular, identificar lo que es común y extender dominios de validez para incluir un conjunto más amplio de casos” (p. 35). De la misma manera, Kaput (1999), entiende por generalización: 
“... extender deliberadamente el rango de razonamiento o comunicación más allá del caso o casos considerados, identificando explícitamente y exponiendo similitud entre casos, o aumentando el razonamiento o comunicación a un nivel donde el foco no son los casos o situación en sí mismos, sino los patrones, procedimientos, estructuras, y las relaciones entre ellos" (p. 136).

Un componente del pensamiento algebraico es el uso del simbolismo algebraico ya que "para caracterizar de forma significativa el pensamiento algebraico no es suficiente ver lo general en lo particular, sino que se debe ser capaz también de expresarlo algebraicamente" (Kieran, 1989, p.165). Pero como señalan English y Warren (1998) hay un desfase entre la habilidad de los estudiantes para reconocer y expresar verbalmente un cierto grado de generalidad y la habilidad para emplear la notación algebraica, pues el uso de esta notación suele plantear dificultades. La generalización de patrones es uno de los contextos en los que es posible empezar a desarrollar formas de pensamiento algebraico. Así, según Radford (2011), "El pensamiento algebraico temprano se considera que está basado en las posibilidades del estudiante para comprender patrones en formas co-variacionales desarrolladas culturalmente y usarlos para tratar con cuestiones de términos lejanos o no especificados" (p. 23).

\subsection{Generalización de patrones}

En las tareas de generalización de patrones se presenta una sucesión de figuras y se pide continuar la sucesión o determinar el número de elementos que componen una figura dada o no especificada; o dado el número de elementos de una figura identificar qué posición ocupa dicha figura en la sucesión. Stacey (1989) distingue aquellas tareas en la que se pide la búsqueda del siguiente término u otro término al cual se pueda llegar mediante recuento, una tabla o un dibujo (generalización cercana) y, por otro, aquellas en los que los procedimientos anteriores resultan laboriosos y es necesario la identificación de un patrón o regla general (generalización lejana).

En este estudio nos centraremos en un caso particular de las tareas de generalización de patrones, las de patrones lineales definidas como:

“aquellos que describen una situación que contiene en el enunciado un dibujo o figura que proporciona los primeros términos $f(1), f(2), f(3), \ldots$ de una progresión aritmética y se pide a los alumnos calcular $f(n)$ para $n$ "pequeño" y para $n$ "grande" y obtener la regla general. El término general viene dado por una función lineal o afin" (Zapatera y Callejo, 2011; p. 600).

Las investigaciones sobre cómo los alumnos de primaria identifican el patrón de una sucesión donde se dan los primeros términos en forma gráfica, han puesto de relieve algunos aspectos importantes del desarrollo del proceso del pensamiento algebraico (Radford, 2011; 2014; Rivera, 2010; Vergel, 2014; Warren, 2005):

- La coordinación entre la estructura espacial y la numérica: Para continuar una sucesión los estudiantes necesitan identificar una regularidad que relacione dos estructuras diferentes: una espacial (distribución espacial de los elementos de las figuras) y otra numérica (número de elementos que componen cada figura).

- La relación funcional: Para identificar un término lejano (o no especificado) se debe establecer una relación funcional entre el término de la sucesión que ocupa la figura y la cantidad de elementos que la forman. 
- El proceso inverso: Para identificar el término de la sucesión que ocupa una figura conocido el número de elementos que la forman, es preciso establecer una relación funcional inversa de la anterior.

Las investigaciones de Radford (2011) con alumnos de $2^{\circ}$ a $4^{\circ}$ grado (de 7 a 10 años) han mostrado que algunos alumnos no llegaban a coordinar las estructuras espacial y numérica de una sucesión formada por figuras, otros sí eran capaces de coordinar las dos estructuras e identificar una regla que permitiera calcular el número de elementos de un término específico, y en grados posteriores algunos estudiantes llegaban a escribir pseudo-fórmulas para expresar la generalidad. Radford interpreta estos resultados desde una perspectiva semiótica subrayando la manera en la que los estudiantes se refieren a lo indeterminado al centrar la atención en cómo expresan la incógnita o variable.

Aunque investigaciones recientes han empezado a mostrar que los estudiantes de los primeros grados son capaces de comprender algunos aspectos de la generalización de patrones antes de introducir el álgebra formal (Cooper \& Warren, 2011; Radford, 2011; Rivera \& Becker, 2011; Vergel, 2014), son necesarias más investigaciones que aporten información sobre las características del pensamiento algebraico temprano y en particular sobre cómo los estudiantes de educación primaria se refieren a lo indeterminado.

\section{Marco teórico}

Para Radford (2010) las formas de pensamiento algebraico permiten comprender las actuaciones (estrategias/procedimientos) de los estudiantes a través de los medios semióticos de objetivación que movilizan cuando se enfrentan a tareas en el contexto de la generalización de patrones. La teoría de la objetivación parte de considerar al individuo no solo como un sujeto pensante sino como un sujeto que vive, piensa y actúa en el marco de una cultura, y de la premisa de que la base de la cognición se encuentra en la praxis social, entendida esta praxis como una actividad humana sensitiva y concreta. Según Radford lo que distingue el pensamiento numérico del algebraico es que este último trata con cantidades indeterminadas o no especificadas como si fueran conocidas y se realizan cálculos con ellas como se hace con los números (analiticidad). Los tres elementos que caracterizan el pensamiento algebraico son: el sentido de indeterminación, la analiticidad y la expresión semiótica de sus objetos.

Desde una perspectiva semiótica Radford (2003) identifica tres formas de pensamiento algebraico: factual, contextual y simbólica. En el pensamiento algebraico factual la generalidad se basa en acciones realizadas sobre números y las actuaciones constan de palabras, gestos y de actividad perceptual. Un ejemplo de esta forma de pensamiento se muestra en la Figura 1 donde se le pregunta a un alumno por el número de círculos que componen la figura 5 de una sucesión (Vergel, 2015, p. 14). Este alumno "en la figura de la izquierda moviliza un gesto indexical señalando los círculos horizontales. La figura del centro muestra el recurso de Esneider del número de círculos horizontales de la figura anterior. Finalmente en la figura de la derecha, se muestra como el estudiante retorna a la figura 5 y hace un deslizamiento para describir la posición y el número de círculos que deben ir en la posición vertical". 


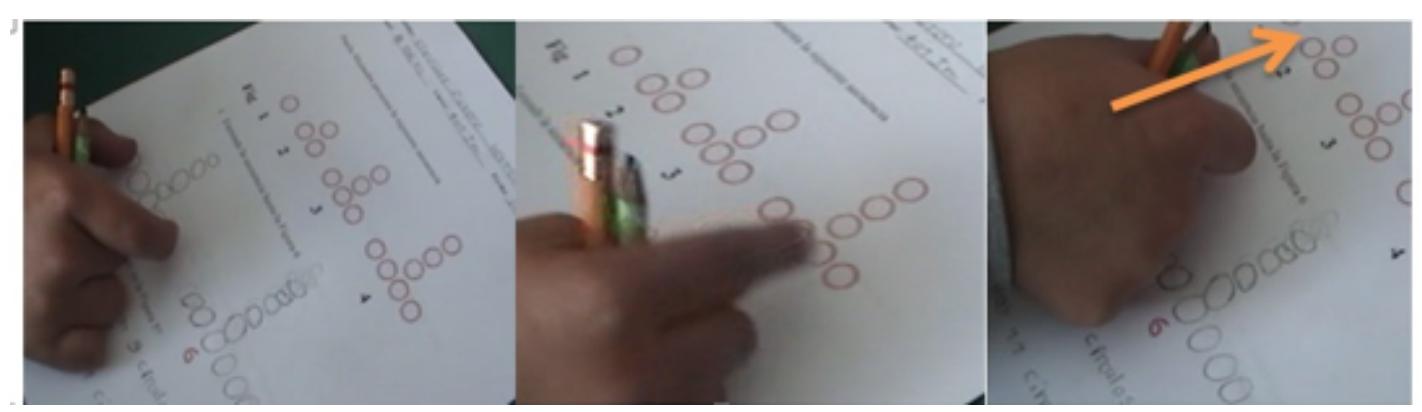

Figura 1. Ejemplo de forma de pensamiento factual. Tomado de Vergel (2015, p. 14)

En el pensamiento algebraico contextual la formulación algebraica es una descripción del término general donde los gestos se sustituyen por términos descriptivos claves, como 'arriba', 'abajo', 'extremos', 'horizontal', 'vertical', etc. Por ejemplo, el número de círculos de cualquier figura de la sucesión del ejemplo anterior se podría expresar de la siguiente forma: en la figura se suman los círculos de abajo, que es igual al número de la figura, con los de arriba, pero arriba hay un círculo menos que abajo. En este caso se utilizan los términos arriba y abajo y en la respuesta subyace el esquema $n+(n-1)$. Finalmente, el pensamiento algebraico simbólico utiliza el simbolismo algebraico, aunque sea incipiente, para expresar la generalidad. El ejemplo de la Figura 2, tomada de Radford (2011) muestra cómo un alumno representa mediante una fórmula lo que escribió: "Necesita poner [tantos cuadrados blancos como] el número de la figura arriba y abajo, más un cuadrado negro arriba”.

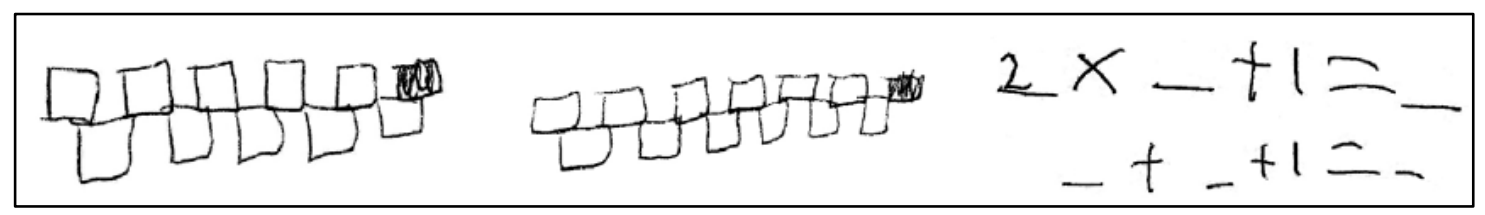

Figura 2. Ejemplo de forma de pensamiento simbólico. Tomado de Radford (2011, p. 187)

Las investigaciones de Radford (2010) muestran que el desarrollo del pensamiento algebraico temprano se mueve desde lo factual a lo contextual y a lo simbólico.

Teniendo en cuenta este marco de referencia y la necesidad de aportar más información acerca de las características del pensamiento algebraico temprano, el objetivo de nuestra investigación es identificar características del pensamiento algebraico de estudiantes de educación primaria (de 6 a 12 años) cuando resuelven problemas de patrones lineales, como un contexto en el que los alumnos deben expresar de alguna manera lo indeterminado. La manera en la que los estudiantes desde $1^{\circ}$ a $6^{\circ}$ curso se refieren a lo indeterminado puede aportar información sobre una trayectoria hipotética de aprendizaje del proceso de generalización en la educación primaria, entendida como un camino hipotético a través del cual el aprendizaje de los estudiantes puede progresar.

Por otra parte, otro aspecto importante que puede aportar información sobre la manera en la que los estudiantes de educación primaria conceptualizan la generalización de patrones lineales tiene que ver con la relación entre las actuaciones (estrategias) de los estudiantes al responder a las cuestiones de generalización lejana y la manera en la que son capaces de invertir este proceso, es decir, averiguar la posición de una figura dado el número de elementos que la componen. De esta manera, nos planteamos las siguientes preguntas de investigación:

- ¿Qué estrategias utilizan los estudiantes de educación primaria para expresar la indeterminación en la generalización lejana? 
- ¿Cómo influye la estrategia utilizada en la generalización lejana en la obtención de la inversión y la regla general?

\section{Método}

A continuación presentamos los participantes, los instrumentos de recogida de datos y el análisis realizado.

\subsection{Participantes}

Los participantes fueron 264 alumnos de educación primaria de un centro público de la provincia de Alicante distribuidos de la siguiente manera: 43 de primer curso, 53 de segundo, 46 de tercero, 41 de cuarto, 35 de quinto y 46 de sexto curso. Estos alumnos no habían recibido ninguna formación específica sobre la generalización de patrones.

\subsection{Instrumentos de recogida de datos}

Los estudiantes de los diferentes cursos resolvieron un problema de generalización de patrones lineales que mantenía siempre la misma estructura (Figura 3). En el enunciado del problema propuesto se dan los tres primeros términos de una sucesión de forma gráfica y se plantean las cuestiones siguientes:

- Cuestión 1: Representar el siguiente término de la sucesión.

- Cuestión 2: Calcular el número de elementos que componen los dos siguientes términos.

- Cuestión 3: Calcular el número de elementos que compone un término lejano.

- Cuestión 4: Identificar cuál es el término que tiene un número dado de elementos.

- Cuestión 5: Identificar la regla general.

En el diseño del instrumento consideramos dos variables: las cuestiones planteadas, y el número usado en la cuestión de generalización lejana. En relación a la primera variable, realizamos dos formatos adaptándolo a la edad de los estudiantes (versión $\mathrm{A}, 1^{\mathrm{o}}$ y $2^{\mathrm{o}}$ curso, y versión $\mathrm{B}$, de $3^{\circ}$ a $6^{\circ}$ curso). En la versión A solo se preguntaban las tres primeras cuestiones -hasta la generalización lejana- y en la versión $\mathrm{B}$ se preguntaban todas las cuestiones. En relación a la segunda variable, el número usado en la cuestión sobre la generalización lejana, era mayor en función del ciclo. En la versión A el número usado fue el 15, y en la versión B el 18. La Figura 3 presenta la versión $\mathrm{B}$ del cuestionario que incluye las cinco cuestiones y se solicita el término 18 en la cuestión de generalización lejana. 


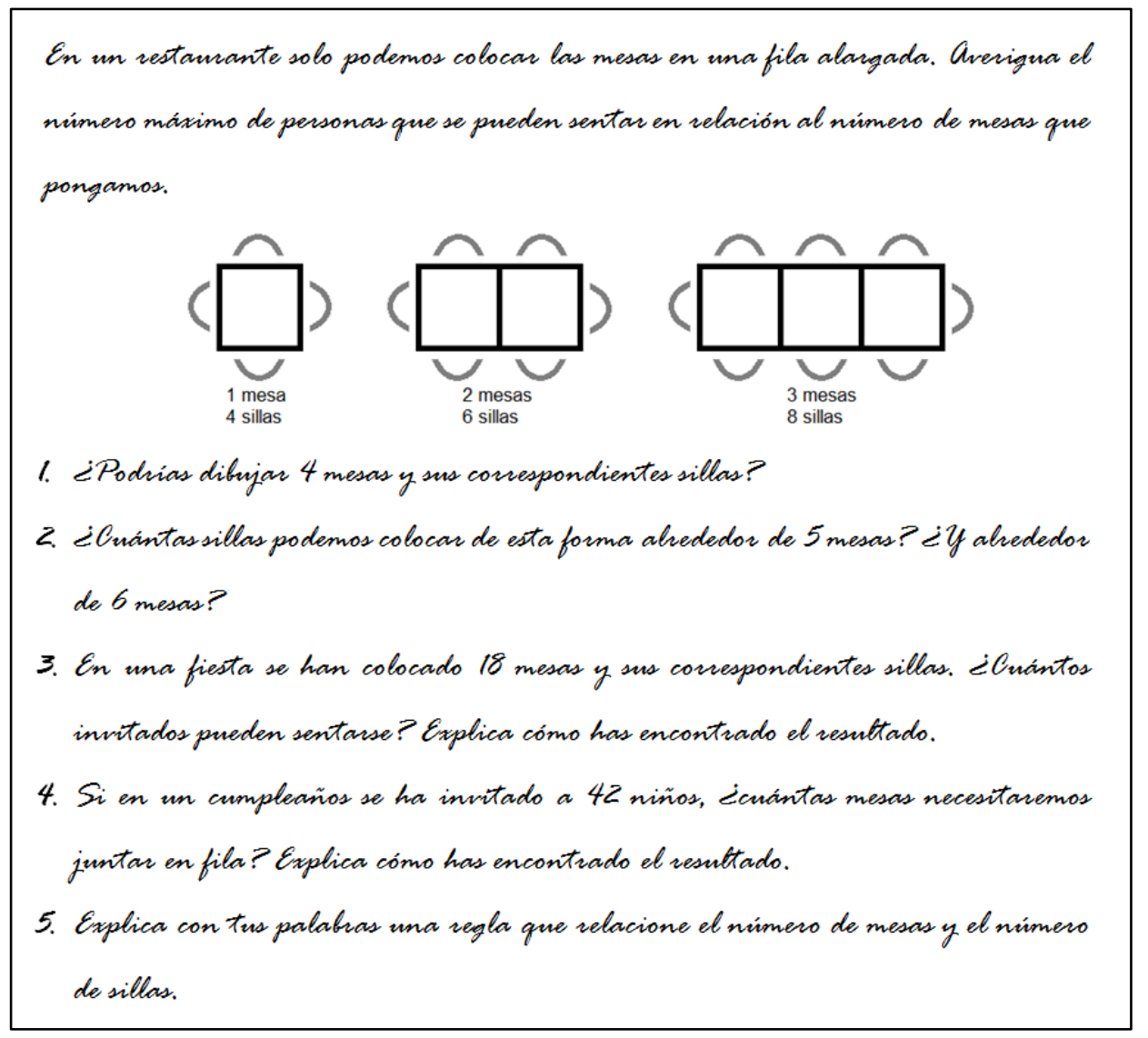

Figura 3. Problema para alumnos de $3^{\circ}$ a $6^{\circ}$ curso

En el problema propuesto se presenta una sucesión de figuras con mesas en fila y con sillas alrededor de las mesas, indicando en los tres primeros términos el número de mesas y de sillas. En las dos primeras cuestiones se pide continuar la sucesión de figuras para 4 mesas y el cálculo de sillas para 5 y 6 mesas respectivamente. La tercera cuestión es de generalización lejana, dado el número de mesas (el término de la sucesión) calcular el número de sillas. En la cuarta cuestión se da el número de sillas y se pide el número de mesas (proceso inverso). En la quinta y última se pide la regla general que relaciona el número de mesas con el de sillas (relación funcional). Las cuatro primeras se refieren a casos particulares y la última al caso general.

Las cuestiones fueron propuestas para indagar sobre aspectos del desarrollo del pensamiento algebraico de los estudiantes puestos de manifiesto al manejar lo indeterminado: la coordinación de las estructuras espacial y numérica, la relación recursiva construyendo un término a partir del anterior, la relación funcional entre el número de mesas y el número de sillas para casos particulares, la relación funcional inversa entre el número de sillas y el número de mesas para casos particulares y la manera en la que los estudiantes desarrollan formas de expresión semiótica de estas relaciones. La articulación de las estructuras espacial y numérica se pone de manifiesto desde la primera cuestión; estas estructuras sirven de referencia para responder a las siguientes cuestiones que a su vez pueden poner de manifiesto otros aspectos del pensamiento algebraico como son la relación funcional y el proceso inverso, así como la manera en la que los estudiantes manejan lo indeterminado. 


\subsection{Análisis}

En este estudio se presenta el análisis de las tres últimas cuestiones. El interés radica en que las respuestas a estas cuestiones permiten indagar sobre las formas de pensamiento algebraico temprano. Este análisis se realizó en dos fases.

En la primera fase se realizó un análisis inductivo en el que tres investigadores realizaron un primer análisis de las respuestas de los estudiantes a la cuestión de generalización lejana obteniendo cuatro categorías de estrategias: (1) representación gráfica o recuento directo sobre un dibujo de la figura pedida, (2) estrategia recursiva o construcción de la sucesión numérica hasta el término buscado añadiendo a cada término la diferencia constante para obtener el siguiente, $a_{n+1}=a_{n}+d$, (3) relación funcional no proporcional, agrupando mentalmente las sillas de la figura pedida y relacionando el número de mesas con el de sillas, asumiendo implícitamente que $f(n)=a n+b, b \neq 0, \mathrm{y}(4)$ razonamiento proporcional, entendiendo implícitamente que hay una relación de proporcionalidad entre el número de mesas y el de sillas, $f(n)=a n$. Estas estrategias muestran distintos aspectos del pensamiento algebraico como son, por una parte, la coordinación entre la estructura espacial y la numérica al representar gráficamente un término particular, la relación funcional al asociar el número de mesas con el de sillas, y el proceso inverso al relacionar el número de sillas con el de mesas; por otra parte, desde una perspectiva semiótica se pueden contemplar distintas formas de expresar la generalidad (Radford, 2003).

Para garantizar la validez y fiabilidad del análisis, cada uno de los investigadores realizó un primer análisis de las respuestas de los estudiantes. A continuación el grupo de investigadores discutió las categorías de estrategias identificadas. Los desacuerdos y acuerdos fueron discutidos con el objetivo de consensuar las categorías y los aspectos del pensamiento algebraico que mostraban las estrategias. Ejemplos de estas estrategias se presentan en el apartado de resultados.

En la segunda fase se relacionaron las estrategias usadas por los estudiantes para responder a la cuestión de generalización lejana (cuestión 3) con el éxito en las cuestiones en que se pedía el número de mesas dado el número de sillas (proceso inverso) (cuestión 4) y en la cuestión en que se pedía la regla general (cuestión 5).

\section{Resultados}

Los resultados se presentan en tres secciones. En primer lugar se muestran las estrategias utilizadas por los estudiantes de primaria en la generalización lejana que evidencian aspectos del pensamiento algebraico. En segundo lugar se muestra el uso de estas estrategias a lo largo de los cursos. En tercer lugar se presenta la relación entre el tipo de estrategia utilizada en la generalización lejana y el nivel de éxito de la inversión y en la obtención de la regla general (de $3^{\circ}$ a $6^{\circ}$ curso).

\subsection{Estrategias utilizadas por los estudiantes que evidencian aspectos del pensamiento algebraico}

Los alumnos utilizaron diferentes tipos de estrategias al intentar responder a la cuestión de generalización lejana que muestran diferentes aspectos del pensamiento algebraico. Presentamos los resultados agrupados por las estrategias utilizadas. 


\subsubsection{Representación gráfica}

La estrategia más frecuente usada por los estudiantes fue hacer una representación gráfica para visualizar el patrón de la secuencia y hacer un recuento directo del número de sillas sobre el dibujo (189 estudiantes- Tabla 1, 71.6\%). De esta manera los estudiantes representaron la relación entre las mesas y las sillas como pedía la cuestión planteada. Un ejemplo de resolución correcta se muestra en la figura 4 (alumno de $2^{\text {do }}$ curso).

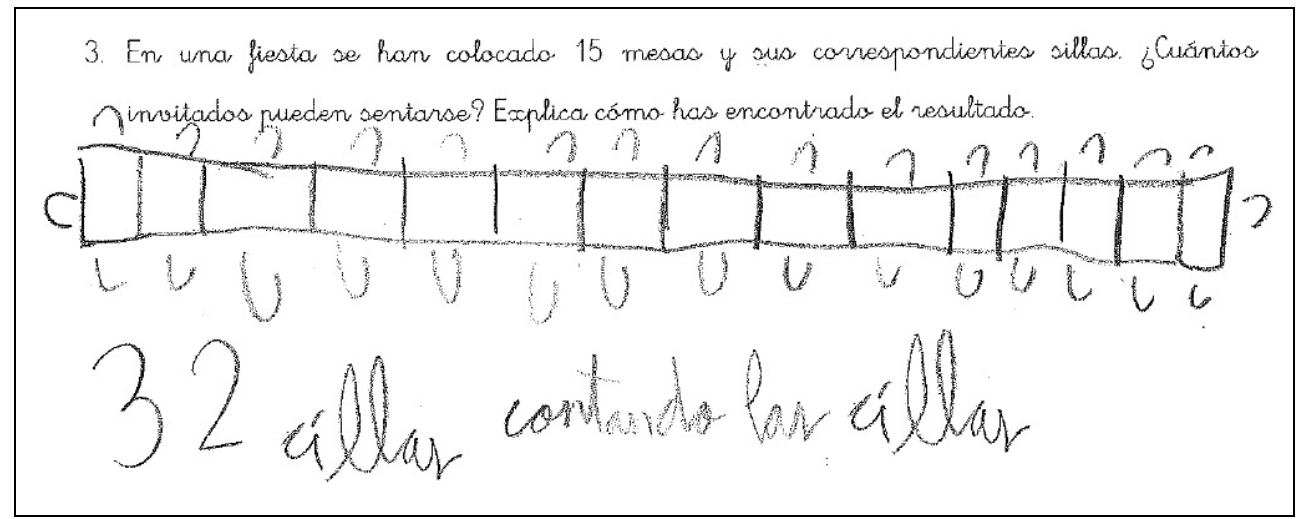

Figura 4. Representación gráfica utilizada por un estudiante de segundo curso.

Esta estrategia pone de manifiesto un aspecto importante del desarrollo del pensamiento algebraico: la coordinación entre las estructuras espacial y numérica.

De los 189 estudiantes, 102 respondieron correctamente (53.9\%), los demás cometieron varios tipos de errores. El más frecuente fue no respetar la estructura espacial ni la estructura numérica (Ejemplo 1, Figura 5). Otros errores fueron respetar la estructura espacial pero no la numérica, pues no representaron el número de mesas pedido (Ejemplo 2, Figura 5 donde el estudiante dibuja 16 mesas en lugar de 15), o respetar la estructura espacial y la numérica pero equivocarse en el conteo (Ejemplo 3, Figura 5).

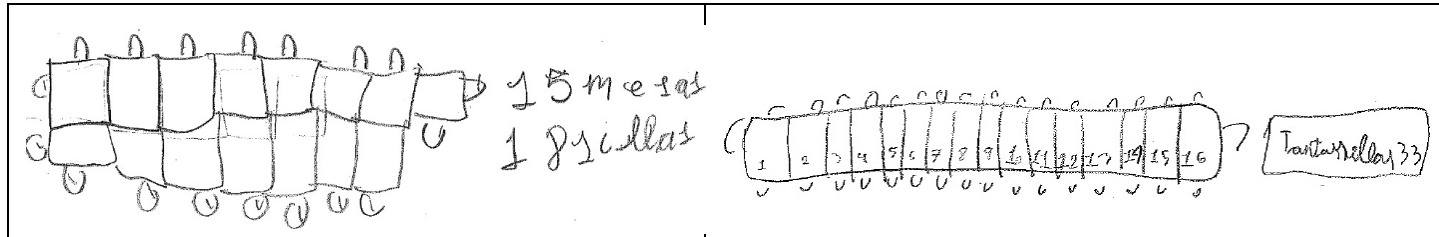

Ejemplo 1 (estudiante $2^{\circ}$ curso) Ejemplo 2 (estudiante $1^{\circ}$ curso)

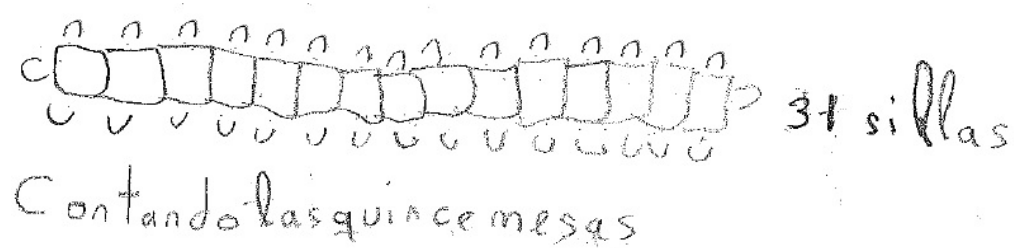

Ejemplo 3 (estudiante $2^{\circ}$ curso)

Figura 5. Ejemplos de resoluciones gráficas que no respetaban la estructura espacial y/o numérica.

\subsubsection{Identificación de la recursividad}


Sólo 3 estudiantes (1.1\%) hallaron el número de sillas haciendo uso del carácter recursivo de la pauta lineal, es decir, sumando 2 sillas por cada mesa añadida. Esta estrategia pone de manifiesto la analiticidad, identificando la diferencia constante de la sucesión y el uso de procedimientos de contar que lo evidencian. La Figura 6 muestra un ejemplo de resolución correcta donde el estudiante construye una tabla.

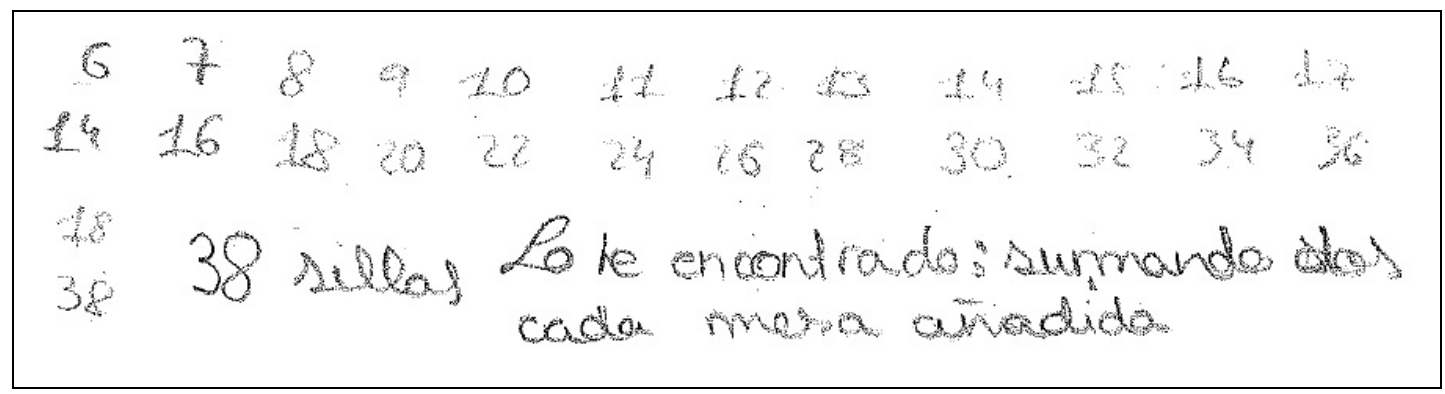

Figura 6. Estrategia recursiva utilizada por un estudiante de sexto curso.

De los 3 estudiantes sólo 1 respondió correctamente, pues los otros dos no identificaron la diferencia constante. Por ejemplo un estudiante de $3^{\text {er }}$ curso (Figura 7), consideró que "cada mesa más es una silla más." Este estudiante usó una manifestación discursiva para dar cuenta de la relación identificada (en este caso equivocada) y consideramos que su respuesta evidencia un incipiente sentido de la indeterminación al referirse a la cantidad indeterminada de mesas como si fueran conocidas; en su expresión subyace el esquema $n+1$ para calcular el número de sillas a partir del término anterior de la sucesión.

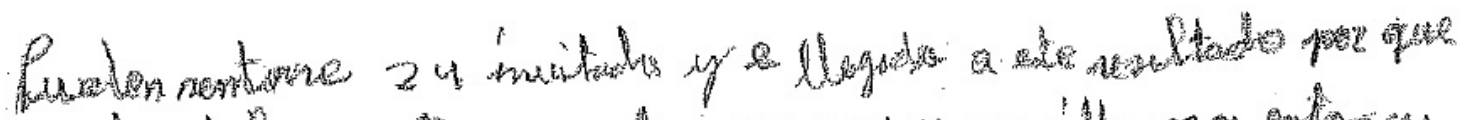

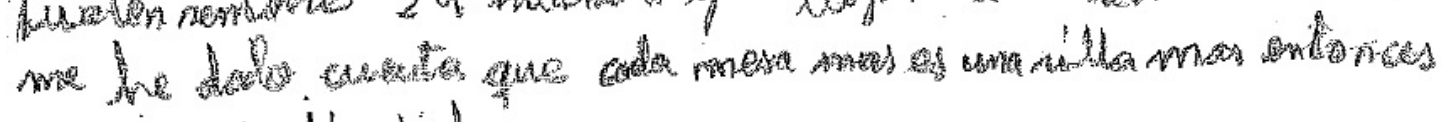

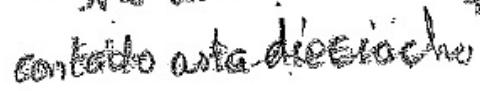

Figura 7. Estrategia recursiva errónea utilizada por un estudiante de tercer curso.

\subsubsection{Relación funcional no proporcional}

43 estudiantes $(16.3 \%)$ identificaron una relación funcional no proporcional entre el número de mesas y el número de sillas usados y realizan los cálculos para determinar el número de sillas en función del número de mesas. La forma en la que los estudiantes evidencian el reconocimiento de la relación funcional entre el número de mesas y sillas ha sido variado, dependiendo de la forma de agrupar las mesas y las sillas. Al explicar los cálculos realizados han utilizado deícticos espaciales con expresiones como arriba, abajo, en medio, extremos, entre otras, que son muestras del pensamiento algebraico temprano en cuanto que más adelante permitirán interpretar el significado de las fórmulas (Vergel, 2014). Además algunos estudiantes al explicar lo que han hecho hablan del número de mesas sin especificar la cantidad, lo que es un indicio más del desarrollo del pensamiento algebraico en cuanto que muestran sentido de la indeterminación al referirse al número de mesas. Esto pone en evidencia la forma de pensamiento algebraico contextual.

Se han observado tres formas de evidenciar el reconocimiento de esta relación y en ellas dos maneras de manifestar el pensamiento algebraico. En la primera forma de 
reconocer la relación los estudiantes multiplican el número de mesas por dos para obtener el número de sillas situadas arriba y abajo, y le suman dos que son las sillas de los extremos (Figura 8). El ejemplo de la Figura 8 pone de manifiesto que el estudiante usa simultáneamente la expresión verbal y los números para dar cuenta de la relación encontrada, pero su expresión verbal va más allá de la descripción de las operaciones realizadas, pues el estudiante no se refiere a las 18 mesas sino que dice "en cada número de mesas", y expresa verbalmente la regla que permite calcular el número de sillas para cualquier número de mesas usando los deícticos arriba, abajo y lados, lo que es una muestra de pensamiento algebraico contextual.

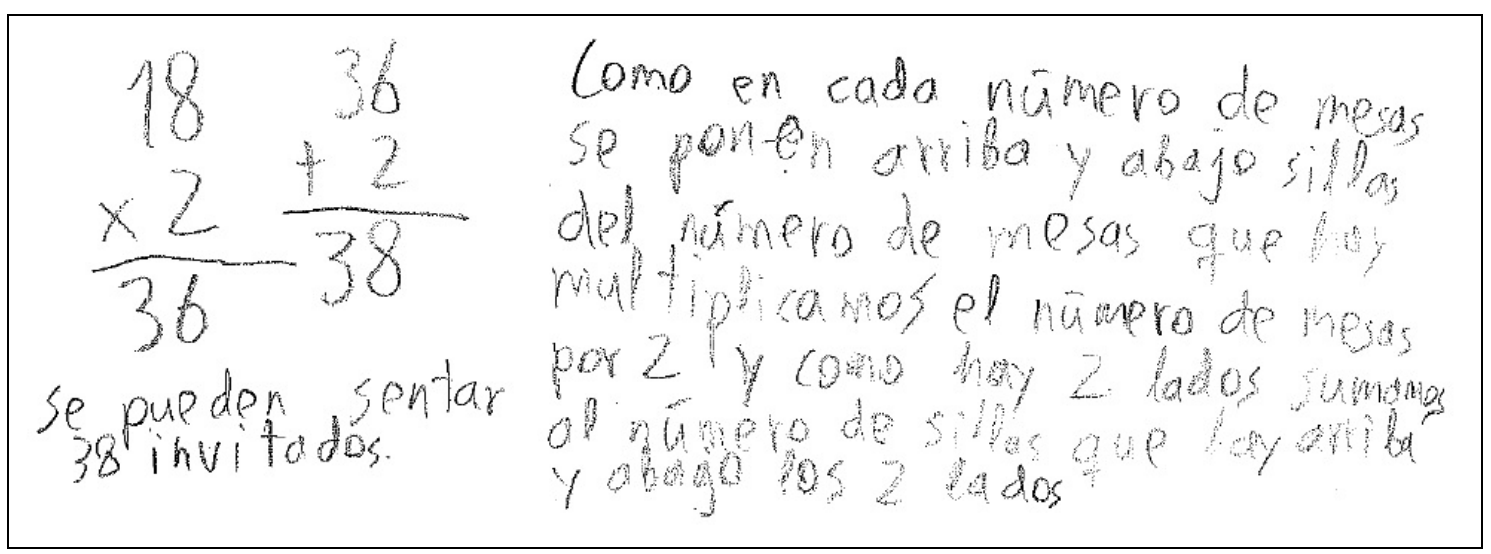

Figura 8. Estrategia funcional tipo 1 utilizada por un estudiante de $4^{\circ}$ curso.

En la segunda forma de reconocer la relación, los estudiantes diferencian entre las mesas de los extremos - con tres sillas-y las de en medio -con dos sillas cada una-, realizan una multiplicación en cada caso y suman los resultados obtenidos (Figura 9). En el ejemplo de la Figura 9 también aparecen los dos modos de representación, numérico y verbal, pero a diferencia del ejemplo anterior el discurso verbal es una simple descripción de las operaciones realizadas y no da muestra de sentido de la indeterminación.

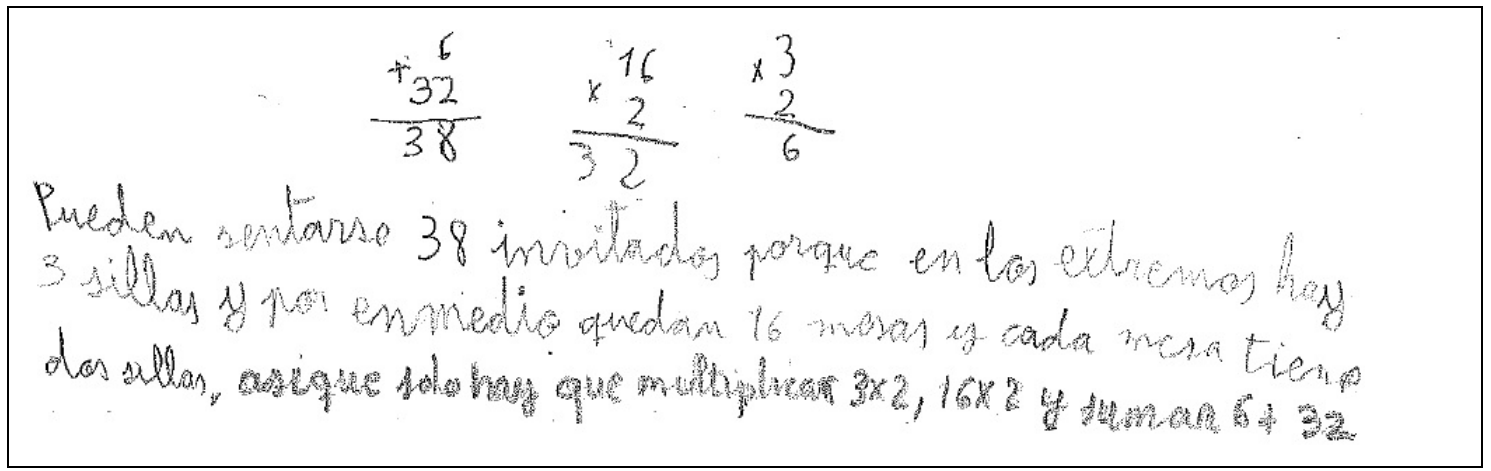

Figura 9. Estrategia funcional tipo 2 utilizada por un estudiante de tercer curso.

En la tercera forma de evidenciar la relación los estudiantes separan una de las mesas de los extremos como si estuviera completa con cuatro sillas, pues le añaden la silla del otro extremo, multiplican el resto de mesas por dos y concluyen realizando la suma (Figura 10). El ejemplo de la Figura 10 también muestra la forma de pensamiento algebraico contextual. 


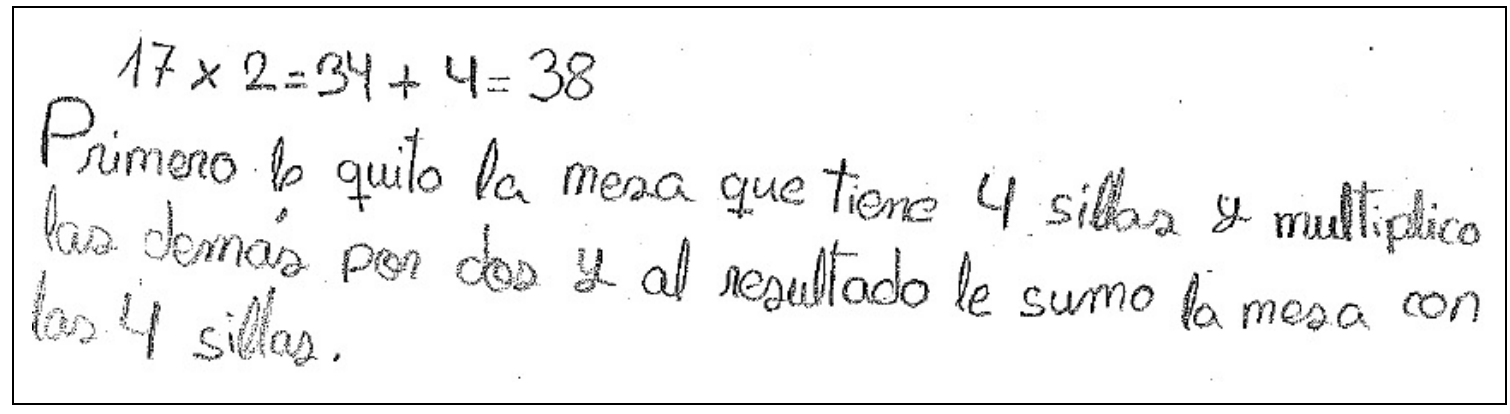

Figura 10. Estrategia funcional tipo 3 utilizada por un estudiante de sexto curso.

Sólo 10 estudiantes que utilizaron la estrategia funcional no obtuvieron éxito (23.2\%). Los errores más frecuentes fueron: identificar que cada mesa tiene dos sillas, pero olvidarse de las sillas de los extremos (Ejemplo 1, Figura 11), o multiplicar por las sillas de los extremos en vez de sumarlas (Ejemplo 2, Figura 11), o sumar una cantidad errónea de sillas de los extremos (Ejemplo 3, Figura 11) o cometer un error de cálculo (Ejemplo 4, Figura 11). Los ejemplos de la Figura 11 no muestran evidencias de pensamiento algebraico contextual, pues los estudiantes se limitan a describir las operaciones realizadas.

\begin{tabular}{|c|c|}
\hline $\begin{array}{c}18 \text { Lo he hechodiciendo } 18 \text { mesas } \\
\frac{2}{36} \text { com he multiplion } 36 \text { invitados } \\
\text { Ejemplo } 1 \text { (estudiante } 4 \text { curso) }\end{array}$ & 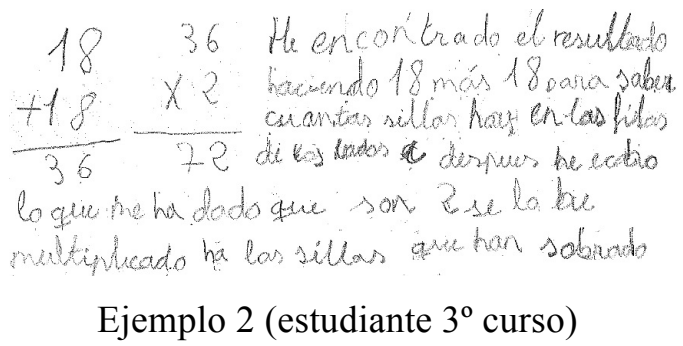 \\
\hline 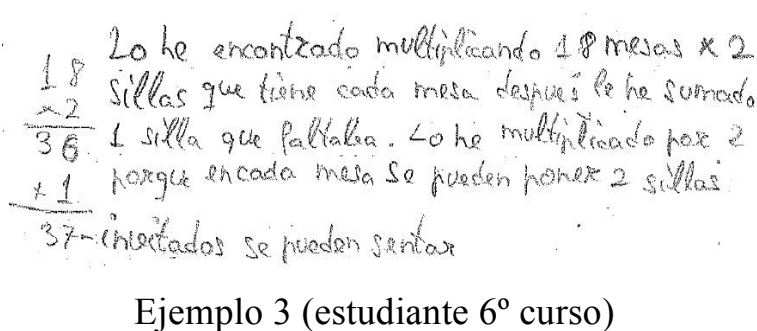 & 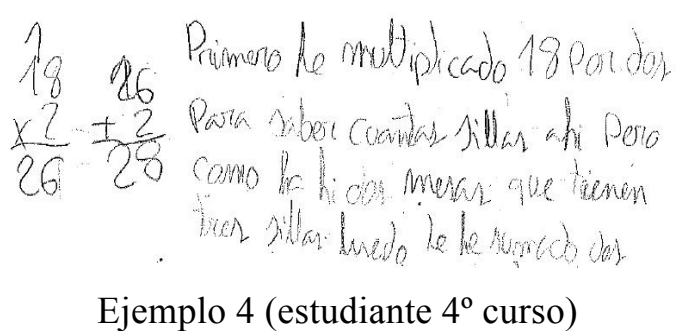 \\
\hline
\end{tabular}

Figura 11. Dificultades en la utilización de la estrategia funcional.

\subsubsection{Estrategia incorrecta usando relaciones de proporcionalidad}

14 estudiantes $(5.3 \%)$ trataron de buscar una relación funcional entre el número de mesas y el de sillas, pero utilizaron un razonamiento proporcional obteniendo así un número erróneo de sillas en relación con el número de mesas. En unos casos multiplicaron por 2 ignorando las sillas de los extremos, y en otro por 3, como el caso del ejemplo de la Figura 12. Estos estudiantes solo se fijaron en una parte de la información proporcionada por el texto y por el dibujo asumiendo una relación de linealidad. 


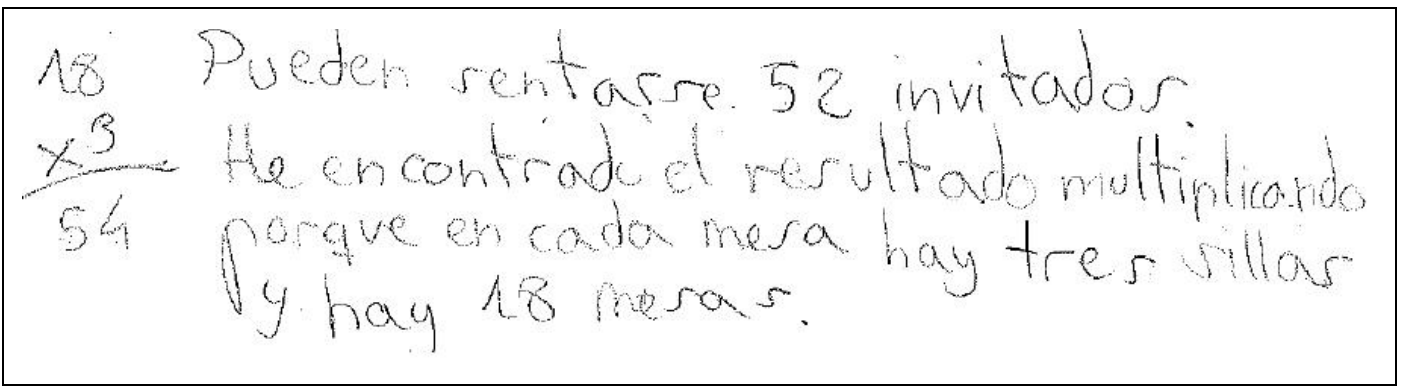

Figura 12. Razonamiento proporcional utilizado por un estudiante de cuarto curso.

Los 15 estudiantes restantes (5.7\%) utilizaron estrategias incorrectas sin sentido o lo dejaron en blanco. Estas estrategias han sido clasificadas como "otras".

\subsection{Uso de las estrategias en la generalización lejana por curso}

La Tabla 1 muestra el uso de las estrategias en la generalización lejana a lo largo de los cursos. El primer número de cada celda corresponde al total de alumnos de educación primaria que utilizó la estrategia, y entre paréntesis se indica el número de estos estudiantes que tuvieron éxito utilizando esa estrategia.

Tabla 1. Nivel de éxito en la generalización lejana (cuestión 3) en función de la estrategia utilizada.

\begin{tabular}{|c|c|c|c|c|c|c|c|}
\hline $\begin{array}{c}\text { Estrategias } \\
\text { utilizadas en la } \\
\text { generalización } \\
\text { lejana } \\
\end{array}$ & $1^{\circ}(\mathrm{N}=43)$ & $2^{\circ}(\mathrm{N}=53)$ & $3^{\circ}(N=46)$ & $4^{\circ}(N=41)$ & $5^{\circ}(N=35)$ & $6^{\circ}(N=46)$ & $\begin{array}{c}\text { Total } \\
(\mathrm{N}=\mathbf{2 6 4})\end{array}$ \\
\hline $\begin{array}{l}\text { Representación } \\
\text { gráfica }\end{array}$ & $40(10)$ & $51(27)$ & $36(19)$ & $25(17)$ & $16(11)$ & $21(18)$ & $189(102)$ \\
\hline Recursiva & $0(0)$ & $0(0)$ & $1(0)$ & $1(0)$ & $0(0)$ & $1(1)$ & $3(1)$ \\
\hline $\begin{array}{l}\text { Funcional no } \\
\text { proporcional }\end{array}$ & $0(0)$ & $0(0)$ & $7(4)$ & $10(7)$ & $10(7)$ & $16(15)$ & $43(33)$ \\
\hline Proporcional & $0(0)$ & $0(0)$ & $1(0)$ & $2(0)$ & $5(0)$ & $6(0)$ & $14(0)$ \\
\hline Otras & $3(0)$ & $2(0)$ & $1(0)$ & $3(0)$ & $4(0)$ & $2(0)$ & $15(0)$ \\
\hline
\end{tabular}

La representación gráfica fue la única estrategia que apareció en todos los cursos y además de manera mayoritaria. Su uso disminuyó de primero a sexto pero aumentó el nivel de éxito, y fue prácticamente la única que usaron los alumnos más jóvenes $\left(1^{\mathrm{er}} \mathrm{y}\right.$ $2^{\text {do }}$ curso). Esta estrategia pone de manifiesto cómo los estudiantes coordinan las estructuras espacial y numérica, como un aspecto del pensamiento algebraico temprano, y se puede observar que esta coordinación va mejorando a lo largo de los años. La disminución del uso de esta estrategia a lo largo de los cursos muestra de qué manera los estudiantes pasan de la representación gráfica de un caso particular a identificar relaciones funcionales que expresan con cálculos específicos y a identificar una regla general que expresan de forma verbal, lo que muestra una forma de pensamiento algebraico contextual, como paso previo para desarrollar el pensamiento algebraico simbólico.

A partir de tercero se amplió la variedad de estrategias apareciendo la recursiva, funcional y proporcional; de ellas la funcional es la que aparece mayoritariamente y su 
uso y nivel de éxito se incrementa de tercer curso a sexto curso. El paso de la representación gráfica, que ilustra la situación del enunciado y se basa en un recuento, a la estrategia funcional que pone en relación dos cantidades mediante operaciones, supone un salto cualitativo importante. Este salto cualitativo se puso de manifiesto en las explicaciones que dieron algunos estudiantes para justificar su respuesta a la cuestión de generalización lejana, pues en lugar de referirse a una figura en particular, la formada por 18 mesas, se referían al número de mesas apareciendo así de forma intuitiva la idea de indeterminación, y usaron deícticos espaciales para describir la posición de las sillas en la regla general como ya hemos visto en algunos ejemplos (Figura 6).

El uso de otras estrategias incorrectas fue mínimo y el uso de la estrategia proporcional fue aumentando a lo largo de los cursos lo que se puede explicar por la aparición en el currículum de contenidos vinculados al razonamiento proporcional.

\subsection{Relación entre la estrategia utilizada en la generalización lejana y la obtención de la inversión y la regla general}

Las cuestiones relativas a la inversión y a la regla general sólo se propusieron a los alumnos de $3^{\circ}$ a $6^{\circ}$ (8 a 12 años). La Tabla 2 muestra la relación entre las estrategias utilizadas en la generalización lejana y el éxito -o no-en las cuestiones donde se pedía identificar el número de mesas dado el número de sillas (cuestión 4, inversión) y la regla general (cuestión 5). También en esta tabla el primer número de cada celda corresponde al total de estudiantes que utilizó esa estrategia en la generalización lejana y el número entre paréntesis el total de ellos que tuvo éxito en la/s cuestión/es indicada/s. En esta tabla no se ha incluido la categoría "otras" pues ningún estudiante que utilizó estrategias incorrectas sin sentido o lo dejó en blanco fue capaz de obtener la inversión o la regla general.

Tabla 2. Niveles de éxito en las cuestiones 4 y 5 en función de la estrategia utilizada en la cuestión 3.

\begin{tabular}{|c|c|c|c|c|c|c|}
\hline $\begin{array}{c}\text { Estrategias utilizadas } \\
\text { en la generalización } \\
\text { lejana }\end{array}$ & $\begin{array}{l}\text { Respuestas } \\
\text { correctas }\end{array}$ & $3^{\circ}(N=46)$ & $4^{\circ}(N=41)$ & $5^{\circ}(N=35)$ & $6^{\circ}(N=46)$ & Total \\
\hline \multirow{3}{*}{$\begin{array}{c}\text { Representación } \\
\text { gráfica }\end{array}$} & $\begin{array}{l}\text { Solo cuestión 4: } \\
\text { Inversión }\end{array}$ & $36(11)$ & $25(2)$ & $16(7)$ & $21(5)$ & $98(25)$ \\
\hline & $\begin{array}{l}\text { Solo cuestión 5: } \\
\text { Regla general }\end{array}$ & $36(0)$ & $25(5)$ & $16(0)$ & $21(4)$ & $98(9)$ \\
\hline & Cuestión 4 y 5 & $36(2)$ & $25(1)$ & $16(2)$ & $21(2)$ & $98(7)$ \\
\hline \multirow{3}{*}{ Recursiva } & $\begin{array}{l}\text { Solo cuestión 4: } \\
\text { Inversión }\end{array}$ & $1(0)$ & $1(0)$ & $0(0)$ & $1(0)$ & $3(0)$ \\
\hline & $\begin{array}{l}\text { Solo cuestión 5: } \\
\text { Regla general }\end{array}$ & $1(0)$ & $1(0)$ & $0(0)$ & $1(0)$ & $3(0)$ \\
\hline & Cuestión 4 y 5 & $1(0)$ & $1(0)$ & $0(0)$ & $1(1)$ & $3(1)$ \\
\hline \multirow{3}{*}{ Funcional } & $\begin{array}{l}\text { Solo cuestión 4: } \\
\text { Inversión }\end{array}$ & $7(2)$ & $10(0)$ & $10(0)$ & $16(1)$ & $43(3)$ \\
\hline & $\begin{array}{c}\text { Solo cuestión 5: } \\
\text { Regla general }\end{array}$ & $7(1)$ & $10(1)$ & $10(2)$ & $16(3)$ & $43(7)$ \\
\hline & Cuestión 4 y 5 & $7(1)$ & $10(4)$ & $10(4)$ & $16(10)$ & $43(19)$ \\
\hline
\end{tabular}




\begin{tabular}{lcccccc}
\hline & $\begin{array}{c}\text { Solo cuestión 4: } \\
\text { Inversión } \\
\text { Polo cuestión 5: }\end{array}$ & $1(0)$ & $2(0)$ & $5(0)$ & $6(0)$ & $14(0)$ \\
Proporcional & $1(0)$ & $2(0)$ & $5(0)$ & $6(3)$ & $14(3)$ \\
& $\begin{array}{c}\text { Regla general } \\
\text { Cuestión 4 y 5 }\end{array}$ & $1(0)$ & $2(0)$ & $5(0)$ & $6(0)$ & $14(0)$ \\
\hline
\end{tabular}

En la Tabla 2 observamos que los estudiantes que usaron una representación gráfica resolvieron con más éxito la cuestión donde se pedía la inversión (32.6\%) que la cuestión donde se pedía la regla general (16.3\%). Esto se puede explicar por el hecho de que el $76.7 \%$ utilizó con éxito la representación gráfica, lo que indica que coordinaban la estructura espacial y la numérica, y esto les pudo ayudar a relacionar el número de sillas con el de mesas apoyándose en el esquema espacial. Sin embargo, la coordinación de estas estructuras no fue suficiente para identificar la regla general ya que para ello hay que identificar una función que relaciona dos cantidades, lo que supone un salto cualitativo importante como ya hemos indicado.

Por otro lado se observa que cuando los estudiantes usaron la estrategia funcional, mayoritariamente resolvieron correctamente la inversión y la regla general (44.2\%).

Los estudiantes que utilizaron una estrategia funcional tuvieron más éxito en ambas cuestiones que aquellos que emplearon una estrategia recursiva (33.3\%) o una representación gráfica (7.1\%). Además, el número de alumnos que usaron una estrategia funcional y tuvieron éxito en ambas cuestiones fue aumentando en relación al curso, pasando de 1 sobre 7 estudiantes en tercero a 10 sobre 16 en sexto; mientras que, con el resto de estrategias, en ningún caso hubo más de 2 estudiantes que tuvieran éxito en ambas cuestiones. Estos datos indican que la identificación de la relación funcional no proporcional, que es un aspecto importante del desarrollo del pensamiento algebraico, sirvió de punto de apoyo para explicitar otras formas de pensamiento algebraico más elaboradas como obtener la relación funcional inversa o la regla general convirtiéndose así en un elemento clave en el desarrollo conceptual de la generalización de patrones lineales en la educación primaria.

Finalmente, los estudiantes que utilizaron para la generalización lejana una estrategia proporcional tuvieron éxito diferente en la inversión y en la regla general. En este caso, tres estudiantes que usaron una estrategia proporcional errónea para la resolución de la generalización lejana pudieron obtener una regla general porque cambiaron a una estrategia funcional dando muestra de la flexibilidad del uso de estrategias.

\section{Discusión y conclusiones}

En este trabajo se identifica características del pensamiento algebraico de alumnos de educación primaria cuando resuelven un problema de generalización de patrones lineales. Hemos analizado en primer lugar las estrategias usadas por los estudiantes de educación primaria en la generalización lejana $\mathrm{y}$, en segundo lugar, hemos caracterizado la influencia de la estrategia utilizada en la generalización lejana para la obtención de la inversión y la regla general. Los resultados obtenidos nos proporcionan características del desarrollo conceptual de la generalización de patrones lineales en educación primaria y los elementos clave en este desarrollo. 


\subsection{Características del desarrollo conceptual de la generalización de patrones lineales en educación primaria}

Las respuestas de los estudiantes a las cuestiones sobre generalización de patrones lineales muestran cómo emergen distintas formas de pensamiento algebraico temprano. Los alumnos en los primeros cursos $\left(1^{\circ}\right.$ y $2^{\circ}$, 6-7 años) llegaron a coordinar las estructuras espacial y numérica. Esta coordinación es un punto de referencia importante para el desarrollo de otras formas de pensamiento algebraico más elaboradas. La coordinación de estas estructuras se puso de manifiesto en el uso casi exclusivo de la representación gráfica para responder a las cuestiones planteadas haciendo el recuento de sillas de las figuras, quizá por influencia de la primera cuestión donde se pedía representar el siguiente término de la sucesión. El uso de una representación gráfica para responder a la cuestión sobre la generalización lejana fue disminuyendo a lo largo de los cursos (del $93.0 \%$ en $1^{\circ}$ al $45.6 \%$ en $6^{\circ}$ ). Al mismo tiempo aumentó el porcentaje de respuestas correctas a lo largo de los cursos (del $25.0 \%$ al $85.7 \%$ ) indicando que los estudiantes eligen otras estrategias y que coordinan mejor las estructuras espacial y numérica.

El cambio de una representación gráfica a una estrategia funcional implica un salto cualitativo en la medida en que los estudiantes relacionan dos cantidades. Al usar esta estrategia los estudiantes se apoyan en la estructura espacial y numérica de la figura, descomponiéndola y recomponiéndola para hacer los cálculos mediante operaciones y no por simple recuento como en el caso de las representaciones gráficas. La explicación de lo que hicieron muestra un modo de pensamiento algebraico más elaborado que puede ser local (cuando dan la explicación del cálculo para números concretos apoyándose en una figura específica, aunque no la dibujen) o una forma de pensamiento algebraico contextual (cuando no dan la explicación sobre una figura particular sino sobre una figura cualquiera, lo que se puso de relieve con el uso de expresiones para referirse a la indeterminación como por ejemplo el número de mesas, y expresando verbalmente la regla general). En ambos casos usaron deícticos espaciales arriba, abajo, los extremos, en medio, para referirse al significado de las operaciones. Esto confirma los resultados de Vergel (2014) con alumnos de $5^{\circ}$ y $6^{\circ}$ grado (11-12 años) sobre formas de pensamiento algebraico temprano. Este autor subraya que esto es una evidencia de la emergencia del pensamiento algebraico temprano en cuanto que:

"en una fórmula algebraica estas expresiones quedan implícitas en su estructura y ésta no deja ver quizás las maneras cómo han evolucionado las formas corpóreas que se han expresado a través de acciones (por ejemplo, gestos, ritmos, miradas, palabras) y que se despliegan en el espacio y el tiempo. Este elemento didáctico es un indicador que nos brinda información valiosa sobre la emergencia del pensamiento algebraico temprano" (Vergel, 2014, p. 184).

Nuestro estudio extiende el de Vergel, pues se ha realizado con alumnos de $1^{\circ}$ a $6^{\circ}$ grado, lo que nos ha permitido constatar tanto manifestaciones primitivas del pensamiento algebraico temprano, por ejemplo la coordinación de la estructura espacial y la numérica puesta de relieve en las representaciones gráficas, como otras más elaboradas como la relación funcional usando deícticos espaciales y en algunos casos sentido de la indeterminación. 
Sin embargo, no siempre los estudiantes tienen éxito en el paso de una representación gráfica a una estrategia funcional, pues a veces usan una estrategia proporcional incorrecta (Stacey, 1989). Esta estrategia aparece en nuestra investigación a partir de tercer curso, que es cuando se introduce en el currículum la proporcionalidad numérica. El uso abusivo de la proporcionalidad en contextos no adecuados ya ha sido descrito en otros estudios (Fernández y Llinares, 2012; Van Dooren, De Bock, Janssens \& Verschaffel, 2008).

Otro aspecto a destacar es que los estudiantes que usaron la representación gráfica resolvieron con más éxito la cuestión donde se pedía la inversión que la cuestión donde se pedía la regla general. Este dato parece indicar que la coordinación de las estructuras espacial y numérica les pudo ayudar a encontrar la relación funcional inversa apoyándose en el esquema espacial; sin embargo la coordinación de estas estructuras no fue suficiente para identificar la regla general. Lo que estos datos parecen indicar es que la posibilidad de generar estrategias alternativas a la representación gráfica de manera espontánea parece ser un indicador del desarrollo conceptual de la generalización de patrones lineales en los estudiantes de educación primaria. Esta inferencia viene apoyada por el hecho de que a partir del tercer curso (alumnos de 8 años) los alumnos utilizaron estrategias funcionales, en unos casos identificando el patrón de la sucesión y en otros no, por ejemplo, considerando una relación proporcional. Este resultado de la relación entre la estrategia usada en la generalización lejana y el éxito para encontrar la regla general o la relación inversa, es una aportación novedosa de nuestra investigación.

\subsection{Elementos clave en el desarrollo conceptual de la generalización de patrones lineales en educación primaria}

La identificación de la relación funcional no proporcional sirvió de punto de apoyo para explicitar otras formas de pensamiento algebraico más elaboradas como obtener la relación funcional inversa o la regla general. Este hecho, permite identificar un salto cualitativo en la forma de proceder de los estudiantes ya que usar, aunque sea de manera implícita, la relación funcional puede ser entendida como un elemento clave en el desarrollo conceptual de la generalización de patrones lineales en educación primaria.

Por tanto, el desarrollo de la estrategia funcional puede ser considerado un elemento clave en la caracterización de una trayectoria de aprendizaje del pensamiento algebraico de los estudiantes de educación primaria en el contexto de la generalización de patrones lineales (Stacey, 1989; NCTM, 2000).

Esta investigación aporta información sobre el desarrollo conceptual de la generalización de patrones lineales de los estudiantes de educación primaria $\left(1^{\circ}\right.$ a $6^{\circ}$ curso). Esta información nos ha permitido identificar características de una trayectoria de aprendizaje de la generalización lineal como un aspecto particular del desarrollo del pensamiento algebraico en alumnos de 6 a 12 años. Esta trayectoria de aprendizaje vincula el uso de estrategias en la resolución de problemas de identificación de patrones lineales y formas de pensamiento algebraico temprano. Este es otro resultado novedoso de nuestro estudio, pues las investigaciones sobre generalización de patrones lineales se han ocupado de estos aspectos por separado, ya que han puesto el énfasis en las estrategias (Stacey, 1989; Warren, 2005) o en las formas de pensamiento algebraico (Radford, 2011; 2014; Vergel, 2014). La conexión entre ambos aspectos tiene 
implicaciones importantes en la enseñanza, pues las estrategias que usan los estudiantes en la resolución de problemas de generalización de patrones lineales evidencian formas de pensamiento algebraico temprano que el maestro debe identificar para favorecer y potenciar esta forma de pensamiento. Nuestro estudio presenta ejemplos de manifestaciones de los estudiantes de educación primaria.

Estos resultados pueden utilizarse en la formación inicial de maestros. Por una parte, porque aportan evidencias de que el pensamiento algebraico se puede desarrollar en la educación primaria. Por otra parte, las producciones de los alumnos pueden servir para diseñar materiales docentes en los programas de formación dirigidos a desarrollar la competencia docente para mirar profesionalmente el pensamiento matemático de los estudiantes (Jacobs, Lamb \& Philipps, 2010; Sánchez-Matamoros, Fernández \& Llinares, 2015). Estos materiales docentes pueden ayudar a que los estudiantes para maestro aprendan a identificar y a describir las estrategias utilizadas por los alumnos de educación primaria cuando resuelven este tipo de problemas y a identificar los elementos matemáticos significativos para el desarrollo del pensamiento algebraico al interpretar las producciones de los estudiantes (Zapatera \& Callejo, 2014).

Por último, nuestro estudio transversal con estudiantes de diferentes edades nos ha permitido observar algunas características del pensamiento algebraico temprano; en futuras investigaciones los resultados obtenidos se podrían contrastar con un estudio longitudinal haciendo el seguimiento de los estudiantes durante un determinado período de tiempo.

\section{Referencias}

Cai, J. \& Knuth, E. (2011) (Ed.). Early algebraization. A global dialogue from multiple perspectives. Berlin: Springer-Verlag.

Carraher, D.W. \& Schliemann, A.D. (2007). Early algebra and algebraic reasoning. En F.K. Lester Jr (Ed.), Second Handbook of Research on Mathematics Teaching and Learning: A Project of the National Council of Teachers of Mathematics (pp. 669-706). Charlotte, NC: Information Age Publishing; Reston VA: National Council of Teachers of Mathematics.

Cooper, T.J. \& Warren, E. (2011). Years 2 to 6 students' ability to generalize: Models, representations and theory for teaching and learning. En J. Cai, J. y E. Knuth, E. (Eds.), Early Algebraization. A Global Dialogue from Multiple Perspectives (187-214). Berlin: Springer-Verlag.

Dreyfus, T. (1991). Advanced mathematical thinking processes. En D. Tall (Ed.), Advanced mathematical thinking (pp. 25-41). Dordtrecht: Kluwer.

English, L.D. \& Warren, E.A. (1998). Introducing the variable through pattern exploration. Mathematics Teacher, 91(2), 166-170.

Fernández, C. \& Llinares, S. (2012). Características del desarrollo del razonamiento proporcional en la Educación Primaria y Secundaria. Enseñanza de las Ciencias, 30(1), 129-142.

Godino, J. D., Aké, L. P., Gonzato, M. \& Wilhelmi, M. R. (2014). Niveles de algebrización de la actividad matemática escolar. Implicaciones para la formación de maestros. Enseñanza de las Ciencias, 32(1), 199-219.

Jacobs, V.R., Lamb, L.C. \& Philipp, R. (2010). Professional noticing of children's mathematical thinking. Journal for Research in Mathematics Education, 41(2), 169-202. 
Kaput, J. (1999). Teaching and learning a new algebra. En Fennema, E. y Romberg, T. A. (Eds.), Mathematics classrooms that promote understanding (pp. 133-155). New Jersey: Lawrence Erlbaum Associates, Inc.

Kieran, C. (1989). The early learning of algebra: A structural perspective. En S. Wagner y C. Kieran (Eds.), Research agenda for mathematics education: Research issues in the learning and teaching of algebra (vol. 4, pp.33-56). Hillsdale, NJ: Erlbaum.

Kieran, C. (2004). Algebraic thinking in the early grades: What is it? The Mathematics Educator, 18(1), 139-151.

NCTM (2000). Principios y Estándares 2000. Reston VA: NCTM. Traducción M. Fernández (Sociedad Andaluza de Educación Matemática Thales) 2003.

Polya, G. (1965). Cómo plantear y resolver problemas. México: Trillas.

Radford, L. (2003). Gestures, speech, and the sprouting of signs: A semiotic-cultural approach to students' types of generalization. Mathematical Thinking and Learning, 5(1), 37-70.

Radford, L. (2010). Algebraic thinking from a cultural semiotic perspective. Research in Mathematics Education, 12(1), 1-19.

Radford, L. (2011). Embodiment, perception and symbols in the development of early algebraic thinking. En Ubuz, B. (Ed.), Proceedings of the 35th Conference of the International Group for the Psychology of Mathematics Education (vol. 4, pp. 17-24). Ankara, Turkey: PME.

Radford, L. (2014). The progressive development of early embodied algebraic thinking. Mathematics Education Research Journal, 26, 257-277.

Rivera, F. D. (2010). Second grade students' preinstructional competence in patterning activity. En Pinto, M. F. y Kawasaki, T. F. (Eds.). Proceedings of the 34th Conference of the International Group for the Psychology of Mathematics Education (vol. 4, pp. 81-88). Belo Horizonte, Brazil: PME.

Rivera, F.D. \& Becker, J.S. (2011). Formation of pattern generalization involving linear figural patterns among middle school students: Results of a three-year study. En J. Cai, J. y E. Knuth, E. (Eds.), Early Algebraization. A Global Dialogue from Multiple Perspectives (pp. 323-366). Berlin: Springer-Verlag.

Sánchez-Matamoros, G., Fernández, C., \& Llinares, S. (2015). Developing pre-service teachers' noticing of students' understanding of the derivative concept. International Journal of Science and mathematics Education, 13(6), 1305-1329.

Socas, M. (2011). La enseñanza del álgebra en la educación obligatoria. Aportaciones de la investigación. Números 77, 5-34.

Stacey, K. (1989). Finding and using patterns in linear generalizing problems. Educational Studies in Mathematics, 20(2), 147-164.

Van Dooren, W., De Bock, D., Janssens, D., \& Verschaffel, L. (2008). The linear imperative: An inventory and conceptual analysis of students' overuse of linearity. Journal for Research in Mathematics Education, 39(3), 311-342.

Vergel, R. (2014). Formas de pensamiento algebraico temprano en alumnos de cuarto y quinto grados de Educación Básica Primaria (9-10 años). (Tesis Doctoral no publicada). Universidad Distrital Francisco José de Caldas, Bogotá, Colombia.

Vergel, R. (2015). ¿Cómo emerge el pensamiento algebraico? Uno, revista de didáctica de las matemáticas, 68, 9-17.

Warren, E. (2005). Young children's ability to generalise the pattern rule for growing patterns. En H.L. Chick y J.L. Vincent (Eds.), Proceedings of the 35th Conference of the 
International Group for the Psychology of Mathematics Education (vol. 4, pp. 305-312). Melbourne: PME.

Zapatera, A. \& Callejo, M. L. (2011). Nivel de éxito y flexibilidad en el uso de estrategias resolviendo problemas de generalización de pautas lineales. En Marín, M., Fernández, G., Blanco, L. y Palarea, M. M. (Eds.), Investigación en Educación Matemática XV (pp. 599610). Ciudad Real: SEIEM.

\section{Agradecimientos.}

Esta investigación ha recibido el apoyo del Proyecto I+D+i EDU2014-54526-R del Ministerio de Ciencia e Innovación, España y de grupos de investigación emergentes GV/2014/075 de la Conselleria de Educación, Cultura y Deporte de la Generalitat Valenciana

\section{Referencias a los autores}

Ma Luz Callejo, Universidad de Alicante (España). 1uz.callejo@ua.es

Álvaro García-Reche, Universidad de Alicante (España).

alvarogarciareche@gmail.com

Ceneida Fernández, Universidad de Alicante (España). ceneida.fernandez@ua.es 


\title{
Early algebraic thinking of primary school pupils (6-12 years) related to linear patterns generalizing problems
}

\author{
Ma Luz Callejo, Universidad de Alicante (España) \\ Álvaro García-Reche, Universidad de Alicante (España) \\ Ceneida Fernández, Universidad de Alicante (España)
}

A main focus of research in mathematics education in recent years has been the development of algebraic thinking in the early grades of education (Radford, 2014) that supports the introduction of early algebra in the primary education curriculum (Cai \& Knuth, 2011; Carraher \& Schliemann, 2007; Godino, Ake, Gonzato \& Wilhelmi, 2014; Socas, 2011). Although recent research has begun to show that students of the early grades are able to understand some aspects of patterns generalization before introducing formal algebra (Cooper \& Warren, 2011; Radford, 2011; Rivera \& Becker, 2011; Vergel, 2014), further investigations are needed to provide characteristics of primary school pupils algebraic thinking when solving linear generalizing problems.

Our research questions are: (i) which strategies do primary school students use to express the indeterminacy in the further generalization? (ii) How does the strategy used in the further generalization influence on obtaining the inversion and the general rule?

The participants were 264 primary school pupils. These participants solved a linear generalizing problem with a succession of figures. We provided them with the first three terms of the succession (graphical representation). Then, they had to answer five questions: (i) a graphical representation of the next term of the succession, (ii) the number of elements of the two next terms, (iii) the number of elements of a further term, (iv) the term that has a given number of elements, and (v) the general rule.

Results show that algebraic thinking starts with the coordination of spatial and numerical structures. It was evidenced in students' use of graphical representation to do a recount. The change from a graphical representation to a functional strategy implies a qualitative step since these students relied on a particular figure to explain their calculations using spatial deictic or they give general explanations of figures using the indeterminacy. Identifying the functional strategy helps students to identify other forms of algebraic thinking more elaborated such as obtaining the inverse functional relationship or the general rule. So, the functional strategy can be considered a key element in the characterization of a primary school students learning trajectory of the algebraic thinking in the context of linear generalization.

These results can be used in teachers training programs to introduce learning trajectories of algebra thinking in the early grades as a reference in teachers' learning of students' mathematical thinking. 